\title{
TIM Seminar
}

\section{Transforming a Desert City into an International Cybersecurity Hub and Ecosystem}

\author{
Roni Zehavi
}

\author{
"In cybersecurity, time is of the essence. We are very") \\ much behind schedule. Collaboration is the only way \\ to catch up, to condense the desperately needed \\ learning and development.
}

Roni Zehavi

CEO, CyberSpark

\section{Overview}

In this special seminar, Roni Zehavi, CEO of CyberSpark (cyberspark.org.il), introduced efforts to build an international cybersecurity hub and ecosystem in Beer-Sheva, Israel. Fifty-four organizations were represented by the attendees, many of whom were from large and small Canadian companies. The purpose of the event was to explore how Canadian and Israeli firms and universities could collaborate to address specific challenges in cybersecurity.

The event was held on April 4, 2016 at Carleton University in Ottawa, Canada, and was jointly hosted by:

- The Technology Innovation Management (TIM; timprogram.ca) program at Carleton University

- The Embassy of Israel in Canada (embassies.gov.il/ottawa/)

- The VENUS Cybersecurity Corporation (venuscyber.com)

\section{Summary}

In introducing the speaker, the Ambassador of Israel to Canada, Rafael Barak, highlighted the potential for collaboration between Israel and Canada to better understand the cyber-threat environment and how countries can cooperate to overcome current and future challenges.

Then, Zehavi (Figure 1) began his seminar by explaining why a cyber-ecosystem is essential, particularly given the scale of the challenges and how little time we have to learn and develop the novel approaches needed to address the challenges posed by social and mobile networks, the Internet of Things, increased sharing of data, etc. He argued that the only way to catch up and condense the required learning into a very short amount of time is for academic organizations, industry, and governments to share information and work together, both within and between countries around the world.

In Israel, the awareness of the cybersecurity challenges and the need to address them is particularly acute. As Zehavi explained, Israel accounts for only $0.1 \%$ of the world's population, but about $10 \%$ of the total global investments in the cybersecurity industry have been

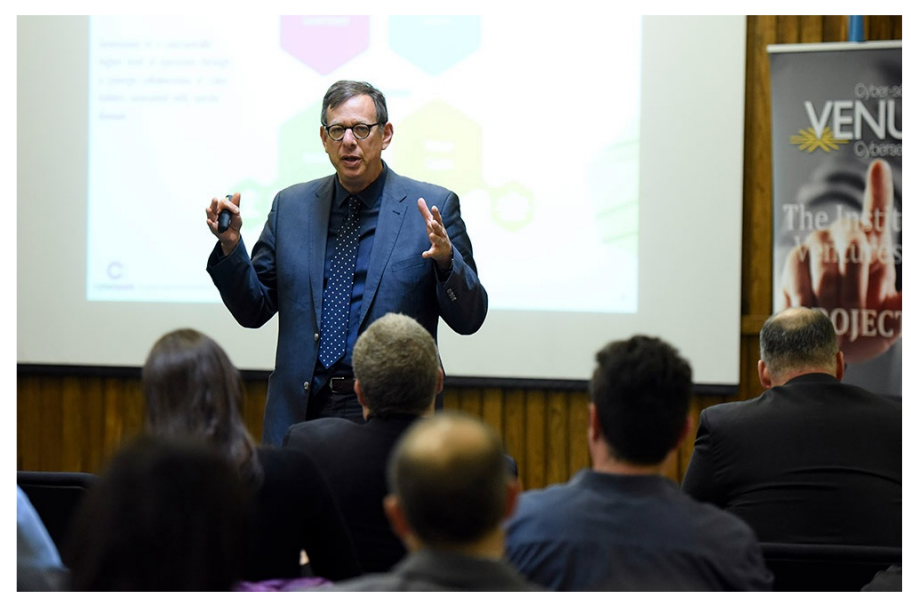

Figure 1. Roni Zehavi, CEO of Cyberspark, introduces the ecosystem in Beer-Sheva, Israel 


\section{Transforming a Desert City into an International Cybersecurity Hub and Ecosystem}

Roni Zehavi

made in the country. Furthermore, the Israeli government has been mounting massive efforts into building the country's cyber-defense capabilities - cybersecurity is recognized as both essential for the protection of the country and a way of re-inventing and re-invigorating its economy. Industry in Israel has also recognized cybersecurity as a new frontier. Israel is home to more than 270 cybersecurity-related companies, 25 of which are multinational companies dealing only with cyberrelated solutions. As Zehavi emphasized, "there really is something going on with cyber in Israel".

Israel's emphasis on cybersecurity is now physically represented by CyberSpark, a high-tech park that also serves as the hub for an ecosystem of international members that collaborate on cybersecurity projects. CyberSpark also refers to the non-governmental organization at the centre of the ecosystem, which is jointly owned by the for-profit members of the ecosystem. Its mission is to "leverage the Israeli cyber-ecosystem into the global cyber-capital centred in Beer-Sheva" to become "unprecedented the world over in terms of innovation and perspective."

To materialize the CyberSpark mission, the ecosystem depends on its four diverse stakeholder groups coming together - literally, as co-located collaborators:

\section{Government}

- the national government plays a substantial role in building and nurturing the ecosystem through a dedicated unit covering all governmental issues

- it coordinates a national effort through investment, research centres, education, regulation, inter-ministry synchronization, benefits policies, profession definitions, tax incentives, etc.

- it operates the educational system upon which the nation and ecosystem depend, and it enables multi-national engagements

- local government also plays a vital role

\section{Industry}

- the ecosystem requires a critical mass of companies, including a mix of small and medium-sized companies and multi-nationals (e.g., Deutsche Telekom, PayPal, Oracle, Lockheed Martin, EMC, and IBM)
- member companies benefit from joint facilities, coopetition (i.e., working together on areas of common benefit, but also competing where they can differentiate), and knowledge sharing (without exposing intellectual property)

\section{Academia}

- the role of academia is primarily represented by BenGurion University of the Negev, Israel's youngest university, which focuses on industry-friendly applied research and includes $\mathrm{PhD}$ studies in cybersecurity. Ben-Gurion physically hosts the CyberSpark hub and its shared research facilities.

- the university benefits from having up-to-date syllabuses coordinated with industry

- the university provides high-quality graduates to the ecosystem and offers accreditation for industry employees

\section{Human capital}

- the ecosystem depends on a critical mass of talents: people who educated, experienced, innovative, creative, motivated, dedicated, and capable of becoming leaders

- the entire spectrum of associated professions is represented, as are all seniority levels

- key inputs of new human capital to the ecosystem are the university and the military

The environment also plays a key role. As examples, Zehavi listed the importance of support from the city and Mayor, the need for facility management, dedicated plans to encourage talent, openness to the needs of the designated audience, a local perspective of a "global city", and a culture that embraces innovation.

As a dedicated execution platform, CyberSpark provides strategy, education, projects, test ranges, an affiliates club, and community events. The platform enables linkages between potential collaborators, offers executive courses on cybersecurity, provides services to enable global players to smoothly transition into the ecosystem. Today, the CyberSpark ecosystem boasts a long list of founders and current tenants, and it continues to grow, in terms of its facilities, membership, and extent of global collaborations. 


\section{Transforming a Desert City into an International Cybersecurity Hub and Ecosystem}

\section{Roni Zehavi}

\section{Natural partnership between Israel and Canada}

Following the presentation, the participants discussed the design and operation of CyberSpark and explored opportunities for collaboration between Israel and Canada. As a starting point, the common ground between the two countries was discussed: in particular, given their first and second rankings among the most educated populations in the OECD (2011), Canadians and Israelis share an understanding of the value of education, research, and innovation. Both countries are at the cutting edge of technology and their academic institutions are some of the best in the world. The two countries also boast strong people-to-people ties with daily flights between Tel Aviv and Toronto, extensive commercial relations supported by a modernized free trade agreement to meet the demands of today's digital economy, and a deep connection through Canada's vibrant Jewish community. In addition, organizations such as the Canada-Israel Industrial Research \& Development Foundation (ciirdf.ca), which was established in 1994, serve as important conduits between small and medium-sized enterprises (SMEs) in both countries that want to forge joint projects in innovation.

Within this context, it was felt that there is a basis for several natural partnerships in cybersecurity between Israel and Canada. Such efforts would build upon a burgeoning network of ties in cybersecurity. For example, several Canadian companies including BlackBerry (blackberry.com) and Magna International (magna.com) have acquired or partnered with Israeli startups. On the other hand, Israelis are reciprocating by looking to Canada for solutions - Israel's Check Point Software Technologies Ltd. (checkpoint.com), one of the top cybersecurity companies in the world, has established offices in Canada to take advantage of the unique skillsets of Canadians. And, just recently, Israel's Magal Security Systems (magal-s3.com) acquired Waterloo's Aimetis (aimetis.com) for $\$ 14$ million.

The audience identified great potential for even more partnerships between companies, research institutions, governmental agencies, and industry associations, especially in key sectors such as banking, telecommunications, energy, transportation, and other critical industries. There was strong interest in strengthening ties between Beer-Sheva and Ottawa, and other Canadian cities, in step with the growth of each country's cybersecurity ecosystems. Institutional arrangements were seen as a key way to spur close connections between entrepreneurs, researchers, and others engaged in finding cybersecurity solutions.

\section{About the Speaker}

Roni Zehavi is the CEO of CyberSpark, the industry initiative created to advance research and development of cyber-solutions in Beer-Sheva, Israel. He has more than 10 years of experience in the entrepreneurial hi-tech arena, integrating highly-innovative and multidisciplinary technologies into sellable products. His range of experience includes stewarding ideas through the development process into the marketplace. His most recent company, "To-BeEducation", is creating a platform upon which teachers and students can upload content that can be transformed into dilemma-based learning games with multiple users, facilitating the development of global learners' communities. An experienced test engineer and pilot from ETPS UK and an Aeronautical Engineer from the Technion, Roni is a well-known expert in the aviation professions, including their operational, methodological, and technological aspects. In 2004, Roni founded Rontal Applications, a leading provider of a 3D-based application for simulations and real-time command-and-control systems. Under his leadership, the company achieved successful results before being acquired by an American corporation.

This report was written by Chris McPhee.

\section{References}

OECD. 2011. Education at a Glance 2011: OECD Indicators. Paris: OECD Publishing.

http://dx.doi.org/10.1787/eag-2011-en

Citation: Zehavi, R. 2016. TIM Seminar - Transforming a Desert City into an International Cybersecurity Hub and Ecosystem. Technology Innovation Management Review, 6(4): 43-45. http://timreview.ca/article/982

Keywords: cybersecurity, CyberSpark, Israel, Canada, collaboration, cooperation 